\title{
Stability of solitary traveling waves of moderate amplitude with non-zero boundary
}

\section{Shan Zheng ${ }^{1 *}\left(0\right.$ and Zhengyong Ouyang ${ }^{2}$}

\section{"Correspondence:}

szheng_math@163.com

'Department of Basic Courses, Guangzhou Maritime Institute, Guangzhou, 510725, P.R. China Full list of author information is available at the end of the article

\section{空 Springer}

\begin{abstract}
Considered here is the stability problem of solitary traveling waves with non-zero boundary of an equation describing the free surface waves of moderate amplitude in the shallow water regime. We employ a transform to convert the stability problem of solitary waves with non-zero boundary to the same one of solitary waves vanishing at infinity for a new equation, such that the abstract stability theorem proposed by Grillakis et al. can work on it. We then show that the solitary traveling waves with non-zero boundary are orbitally stable under certain parameter conditions.
\end{abstract}

MSC: 35Q51; 35A35; 35B30; 35G25

Keywords: moderate amplitude; solitary traveling waves with non-zero boundary; orbital stability

\section{Introduction}

Constantin and Lannes [1] derived an equation to describe surface waves of moderate amplitude in the shallow water regime,

$$
u_{t}+u_{x}+6 u u_{x}-6 u^{2} u_{x}+12 u^{3} u_{x}+u_{x x x}-u_{x x t}+14 u u_{x x x}+28 u_{x} u_{x x}=0,
$$

which is based on an earlier equation proposed by Johnson [2] and arises as an approximation of the Euler equations. On the explanation of moderate amplitude, we refer to Refs. [1] and [3]. The equations (e.g., Camassa-Holm equation) describing moderate amplitude water waves capture a wider range of nonlinear phenomena than those (e.g., KdV equation) with small amplitude. Firstly, we state some results on the well-posedness of equation (1.1). In [1], the local well-posedness of (1.1) with initial data in $H^{s}(R)\left(s>\frac{5}{2}\right)$ was proved. Using the pseudoparabolic regularization technique, Lai and Wu [4] established its local well-posedness in the Sobolev space $H^{s}(R)$ with $s>\frac{3}{2}$ via a limiting procedure. As well by applying Kato's [5] semigroup approach to quasi-linear equations, Duruk and Geyer [6] improved the result on the local well-posedness to $H^{s}(R)$ with $s>\frac{3}{2}$. By the same method, Duruk [7] proved that this result also holds true for the corresponding spatially periodic Cauchy problem. In [8], Zhou and Mu obtained a semigroup of global conservative solutions, which depend continuously on the initial data. Furthermore, by employing Littlewood-Paley decomposition and transport equation theorem, $\mathrm{Mi}$ and $\mathrm{Mu}$ [3] extended the local well-posedness of (1.1) in Sobolev spaces $H^{s}(R)\left(s>\frac{3}{2}\right)$ to Besov spaces $B_{p, r}^{s}(R)$ with $p, r \in[1, \infty), s>\max \left\{\frac{3}{2}, 1+\frac{1}{p}\right\}$ and critical case with $s=\frac{3}{2}, p=2$ and $r=1$.

(c) The Author(s) 2017. This article is distributed under the terms of the Creative Commons Attribution 4.0 International License (http://creativecommons.org/licenses/by/4.0/), which permits unrestricted use, distribution, and reproduction in any medium, provided you give appropriate credit to the original author(s) and the source, provide a link to the Creative Commons license, and indicate if changes were made. 
The following are some results on the existence and stability of solitary waves for (1.1). Geyer [9] showed the existence of solitary traveling wave solutions for (1.1) and captured some qualitative features of the solitary waves. Duruk and Geyer [10] proved that the solitary traveling waves are orbitally stable by using an approach relying on the method proposed by Grillakis et al. [11] and Constantin [12]. In [13], Gausull and Geyer further studied traveling waves of equation (1.1) and established the existence of periodic waves, compactons and solitary waves under some parameter conditions. Here we need to point out that they proved the existence of solitary waves tending to a non-zero constant at infinity. However, the orbital stability of such solitary waves with non-zero boundary for (1.1) has not been studied yet.

In this paper, we investigate the stability problem of solitary waves with non-zero boundary for (1.1). Since the method proposed by Grillakis et al. [11] cannot be directly used to deal with it, we make a translation transformation to reduce the stability problem of such solitary waves to the same problem of solitary waves vanishing at infinity for a new equation, such that the method in $[10,11]$ and [12] can work on it. The rest of this paper is organized as follows. In the next section, we study the solitary waves with non-zero boundary, give the definition of orbital stability and establish local well-posedness of the associated equation. In Section 3 we give the Hamiltonian structure and conservation laws. Finally, we prove the stability of solitary waves with non-zero boundary in Section 4 .

\section{Solitary waves and local well-posedness}

\subsection{Solitary waves with nonvanishing boundary}

This subsection is mainly concerned with a solitary wave solution tending to a non-zero constant at infinity of (1.1). Recalling Proposition 2.3 and Proposition 3.2 in [13], we see that, under some parameter conditions, there exist both positive and negative solitary waves with nonvanishing boundary, whose amplitude is strictly increasing or decreasing with wave speed $c$. But the main goal of this paper is to prove that a solitary wave solution with non-zero boundary could be orbitally stable. Therefore, throughout this paper, we only consider the case in which the solitary wave solutions are positive, tend to a non-zero constant at infinity, and their amplitude is strictly increasing with wave speed $c$.

Let $u(x, t)=\varphi(x-c t)$ be such a solitary traveling wave solution with non-zero boundary of (1.1) (see Figure 1), whose existence was proved in [10], the solitary wave $\varphi(x-c t)$ tends to a constant $k$ at infinity. When $k=0$, the stability problem of solitary waves was solved in [10]. In the case of $k \neq 0$, we introduce the transformation $\varphi(x-c t)=\phi(x-c t)+k$. Substituting it into (1.1), we have that $\phi(x-c t)$ is a solitary wave solution vanishing at infinity of the following equation:

$$
v_{t}+p_{1}(k) v_{x}+p_{2}(k) v v_{x}+p_{3}(k) v^{2} v_{x}+12 v^{3} v_{x}+p_{4}(k) v_{x x x}-v_{x x t}+14 v v_{x x x}+28 v_{x} v_{x x}=0,
$$

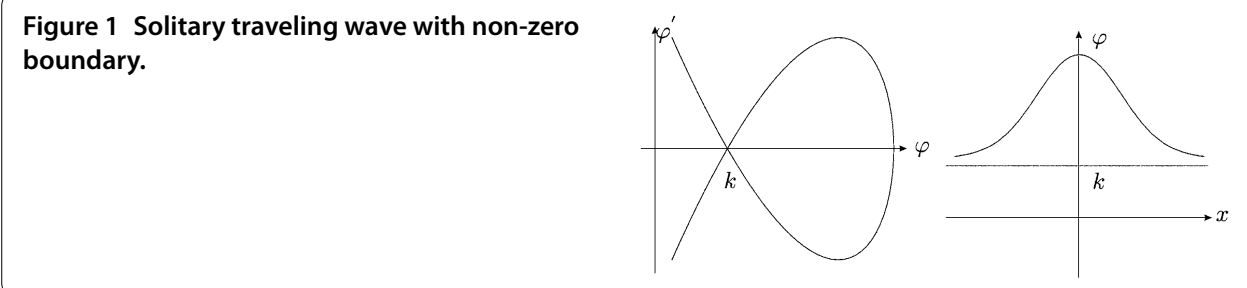


with

$$
\begin{aligned}
& p_{1}(k)=1+6 k-6 k^{2}+12 k^{3}, \\
& p_{2}(k)=6-12 k+36 k^{2}, \\
& p_{3}(k)=36 k-6, \\
& p_{4}(k)=1+14 k .
\end{aligned}
$$

Now, via integration, we compute to find some equalities for $\phi$, which will be used in the later proof of the stability.

Let $\phi(\xi)=\phi(x-c t)$ and substituting it into (2.1) gives

$$
\begin{aligned}
& -c \phi^{\prime}+p_{1}(k) \phi^{\prime}+p_{2}(k) \phi \phi^{\prime}+p_{3}(k) \phi^{2} \phi^{\prime}+12 \phi^{3} \phi^{\prime}+p_{4}(k) \phi^{\prime \prime \prime}+c \phi^{\prime \prime \prime} \\
& +14 \phi \phi^{\prime \prime \prime}+28 \phi^{\prime} \phi^{\prime \prime}=0,
\end{aligned}
$$

where the prime denotes the derivative of $\phi$ with respect to $\xi$.

Integrating (2.2) once yields

$$
\left(p_{1}(k)-c\right) \phi+\frac{p_{2}(k)}{2} \phi^{2}+\frac{p_{3}(k)}{3} \phi^{3}+3 \phi^{4}+\left(p_{4}(k)+c+14 \phi\right) \phi^{\prime \prime}+7\left(\phi^{\prime}\right)^{2}=0,
$$

where the integral constant equals zero since $\phi, \phi^{\prime}, \phi^{\prime \prime} \rightarrow 0$ as $|\xi| \rightarrow \infty$.

Multiplying both sides of (2.3) by $\phi^{\prime}$ and integrating once again can lead to

$$
\left(\phi^{\prime}\right)^{2}=\phi^{2} \frac{c-p_{1}(k)-\frac{p_{2}(k)}{3} \phi-\frac{p_{3}(k)}{6} \phi^{2}-\frac{6}{5} \phi^{3}}{c+p_{4}(k)+14 \phi} .
$$

Since $\varphi(x-c t)=\phi(x-c t)+k$ is a solitary traveling wave solution tending to $k$ at infinity of (1.1), then $\phi(x-c t)$ is the solitary wave approaching zero of (2.1). Conversely, if $v(x, t) \in$ $C\left([0, T), H^{2}(R)\right)$ is a solution of $(2.1)$, then $u(x, t)=v(x, t)+k$ is the solution tending to $k$ at infinity to (1.1).

Remark 2.1 According to Proposition 2.3 and Proposition 3.2 in [13], we choose the traveling wave solution $u(x, t)=\varphi(x-c t) \rightarrow k(|x-c t| \rightarrow \infty)$ of (1.1) whose amplitude is strictly increasing with the wave speed $c$, to prove the associated stability problem. As in the above analysis, via a translation transform, the solitary wave solution $\phi(x-c t)$ of $(2.1)$, corresponding to $\varphi(x-c t)$, vanishes at infinity, and its amplitude is also strictly increasing with the wave speed $c$. It should be noted that the monotonicity plays an important role in the proof of orbital stability below.

\subsection{Definition of orbital stability}

As already observed by Benjamin and coworkers [14,15], a solitary wave cannot be stable in the strictest sense of the word. To understand this, consider two solitary waves of different height, centered initially at the same point. Since the two waves have different amplitudes, they have different velocities. As time passes, the two waves will apart, no matter how small the initial difference was. However, in the situation just described, it is evident that two solitary waves with slightly differing height will stay similar in shape during the time 
evolution. Measuring the difference in shape, therefore, will give an acceptable notion of stability. This sense of orbital stability was introduced by Benjamin [14]. We say a solitary wave is orbitally stable if a solution $u$ of equation (1.1) that is initially sufficiently close to a solitary wave will always stay close to a translation of the solitary wave during the time of evolution. Based on the analysis above and in Section 2.1, we give the definition of orbital stability for the solitary wave with nonvanishing boundary.

Definition 2.1 A solitary wave $\varphi(x-c t) \rightarrow k(|x-c t| \rightarrow \infty)$ of (1.1) is called orbitally stable if for every $\epsilon>0$ there exists $\delta>0$ such that: if $u(x, t)=v(x, t)+k$ is a solution of (1.1) with $v(x, t) \in C\left([0, T), H^{2}(R)\right)(0<T \leq \infty)$ satisfying $\|u(x, 0)-\varphi(x)\|_{H^{2}}<\delta$, then for every $t \in[0, T)$ we have

$$
\inf _{\eta \in R}\|u(x, t)-\varphi(x-c t-\eta)\|_{H^{2}}<\epsilon .
$$

Remark 2.2 Although $u(x, t)$ and $\varphi(x-c t)$ do not belong to $H^{2}(R)$, Definition 2.1 is meaningful since $\|u(x, 0)-\varphi(x)\|_{H^{2}}=\|v(x, 0)-\phi(x)\|_{H^{2}}$ and $\|u(x, t)-\varphi(x-c t-\eta)\|_{H^{2}}=$ $\|v(x, t)-\phi(x-c t-\eta)\|_{H^{2}}$, where $v(x, t), \phi(x-c t-\eta) \in H^{2}(R)$ for any given $t$. Essentially, we only need to deal with the stability problem of $\phi(x-c t)$ for equation $(2.1)$.

\subsection{Local well-posedness}

To prove the stability of solitary waves, the well-posedness for equation (2.1) is firstly required. The Cauchy problem of (2.1) can be rewritten as

$$
\begin{aligned}
& v_{t}=A(v) v+f(v), \\
& v(x, 0)=v_{0}(x),
\end{aligned}
$$

where

$$
\begin{aligned}
& A(v)=\left(p_{4}(k)+14 v\right) \partial_{x} \\
& f(v)=-\left(1-\partial_{x}^{2}\right)^{-1}\left[\left(p_{1}(k)+p_{4}(k)\right) v_{x}+\left(7+\frac{p_{2}(k)}{2}\right) v^{2}+\frac{p_{3}(k)}{3} v^{3}+3 v^{4}+14 v_{x}^{2}\right] .
\end{aligned}
$$

With the same proceeding as the proof of Theorem 3.1 in [6], we have the following lemma on local existence of (2.1).

Lemma 2.1 ([6]) Let $v_{0} \in H^{s}(R)(s>3 / 2)$. Then there exists $T>0$, depending on $v_{0}$, such that there is a unique solution $v(t, x)$ to (2.6) and (2.7) satisfying $v(t, x) \in C\left([0, T), H^{s}(R)\right) \cap$ $C^{1}\left([0, T), L^{2}(R)\right)$.

Another way to prove the local well-posedness of (2.1) is the application of LittlewoodPaley decomposition and transport equation theory as in [3]. The local well-posedness of (2.1) can be established in Besov spaces.

Lemma 2.2 ([3]) Let $p, r \in[1, \infty]$ and $s>\max \{3 / 2,1+1 / p\}$. Assume that $v_{0} \in B_{p, r}^{s}(R)$. Then there exist a time $T>0$ and a unique solution $v \in C\left([0, T] ; B_{p, r}^{s}(R)\right) \cap C^{1}\left([0, T] ; B_{p, r}^{s-1}(R)\right)$ to the Cauchy problem (2.6) and (2.7). 
Remark 2.3 In Lemma 2.2, when $p=r=2$, the Besov spaces $B_{p, r}^{s}(R)$ are namely the Sobolev spaces $H^{s}(R)$. The proof of Lemmas 2.1 and 2.2 is the same as in [6] and [3], we omit the details here.

\section{Hamiltonian system and conservation laws}

Equation (2.1) can be rewritten as the following Hamiltonian system:

$$
v_{t}=J F^{\prime}(v)
$$

where $J=-\left(1-\partial_{x}^{2}\right)^{-1} \partial_{x}$ is a skew-symmetric linear operator, the prime denotes variational derivative of $F(v)$, and

$$
F(v)=\int_{R}\left(\frac{p_{1}(k)}{2} v^{2}+\frac{p_{2}(k)}{6} v^{3}+\frac{p_{3}(k)}{12} v^{4}+\frac{3}{5} v^{5}-\frac{p_{4}(k)}{2} v_{x}^{2}-7 v v_{x}^{2}\right) \mathrm{d} x
$$

is a functional of $v$.

Another functional of $v$ is given by

$$
E(v)=\frac{1}{2} \int_{R}\left(v^{2}+v_{x}^{2}\right) \mathrm{d} x
$$

which can be understood as the kinetic energy of the waves. Both quantities $E(v)$ and $F(v)$ are critically important to the proof of stability of solitary waves, which are showed to be conserved by the following lemma.

Lemma 3.1 The functionals $E(v)$ and $F(v)$ defined above are conserved quantities under equation (2.1).

Proof Multiplying (2.1) by $v$ and integrating over $R$, we have

$$
\frac{\mathrm{d}}{\mathrm{d} t}\left(\frac{1}{2} \int_{R}\left(v^{2}+v_{x}^{2}\right) \mathrm{d} x\right)=\frac{\mathrm{d}}{\mathrm{d} t} E(v)=0
$$

To show $F(v)$ is invariant with respect to $t$, we need to use the skew symmetry of the operator $J$ in equation (3.1). It follows from equation (3.1) that

$$
\frac{\mathrm{d}}{\mathrm{d} t} F(v(t))=\left(F^{\prime}(v), v_{t}\right)=\left(F^{\prime}(v), J F^{\prime}(v)\right)=-\left(J F^{\prime}(v), F^{\prime}(v)\right)
$$

It follows that

$$
\frac{\mathrm{d}}{\mathrm{d} t} F(v(t))=0
$$

\section{Orbital stability}

The method used to verify this type of stability is attributed to Grillakis, Shatah and Strauss [11], and we essentially apply a theorem presented therein to deal with it. For this purpose, we list the following assumptions: 
(A1) For every $v_{0} \in H^{s}(R)(s>3 / 2)$, there exists a solution $v(t, x) \in C\left([0, T) ; H^{s}(R)\right) \cap C^{1}\left([0, T) ; H^{s-1}(R)\right)$ of $(2.1)$ with $v(0, x)=v_{0}$ for some $T>0$. Furthermore, there exist functionals $E(v)$ and $F(v)$ such that they are conserved for solutions of (2.1).

(A2) For every $c \in\left(c_{1}, c_{2}\right)$, there exists a traveling wave solution $\phi \in H^{2}(R)$ of (2.1), where $\phi>0$ and $\phi_{x} \not \equiv 0$. The mapping $c \rightarrow \phi(x-c t)$ is $C^{1}\left(\left(c_{1}, c_{2}\right) ; H^{2}(R)\right)$. Moreover, $\phi$ satisfies $c E^{\prime}(\phi)-F^{\prime}(\phi)=0$, where $E^{\prime}$ and $F^{\prime}$ are the variational derivatives of $E$ and $F$, respectively.

(A3) For every $c \in\left(c_{1}, c_{2}\right)$, the linearized Hamiltonian operator around $\phi$ defined by

$$
H_{c}: H^{1}(R) \rightarrow H^{-1}(R), \quad H_{c}=c E^{\prime \prime}(\phi)-F^{\prime \prime}(\phi)
$$

has exactly one negative simple eigenvalue, its kernel is spanned by $\phi_{x}$ and the rest of its spectrum is positive and bounded away from zero.

Theorem 4.1 ([11]) Under assumptions (A1), (A2) and (A3), a solitary wave solution $\phi(x-$ ct) of (2.1) is stable if and only if the scalar function $d(c)=c E(\phi)-F(\phi)$ is convex in a neighborhood of $c$.

Firstly we verify that equation (2.1) satisfies assumptions (A1)-(A3). Assumption (A1) is guaranteed by Lemmas 2.1 and 2.2 .

To prove assumptions (A2) and (A3), we need to calculate the variational derivatives of functionals $E(v)$ and $F(v)$.

$$
\begin{aligned}
& E^{\prime}(v)=\left(1-\partial_{x}^{2}\right) v, \\
& E^{\prime \prime}(v)=1-\partial_{x}^{2}, \\
& F^{\prime}(v)=p_{1}(k) v+\frac{p_{2}(k)}{2} v^{2}+\frac{p_{3}(k)}{3} v^{3}+3 v^{4}+p_{4}(k) v_{x x}+7 v_{x}^{2}+14 v v_{x x}, \\
& F^{\prime \prime}(v)=p_{1}(k)+p_{2}(k) v+p_{3}(k) v^{2}+12 v^{3}+p_{4}(k) \partial_{x}^{2}+14 v_{x x}+14 v \partial_{x}^{2}+14 v_{x} \partial_{x} .
\end{aligned}
$$

From the above first order variational derivatives of $E(v)$ and $F(v)$, we know that equation (2.3) can be rewritten as

$$
c E^{\prime}(\phi)-F^{\prime}(\phi)=0 .
$$

Thus assumption (A2) is ensured.

By a direct calculation we obtain the following linearized operator $H_{c}$ :

$$
\begin{aligned}
H_{c} & =c E^{\prime \prime}(\phi)-F^{\prime \prime}(\phi) \\
& =-\partial_{x}\left(\left(c+p_{4}(k)+14 \phi\right) \partial_{x}\right)+c-p_{1}(k)-p_{2}(k) \phi-p_{3}(k) \phi^{2}-12 \phi^{3}-14 \phi_{x x} .
\end{aligned}
$$

Since $H_{c}$ is a second order linear differential operator, the corresponding spectrum equation $H_{c} v=\lambda v$ can be written as the Sturm-Liouville problem

$$
-\left(p v_{x}\right)_{x}+(q-\lambda) v=0
$$

where $p=c+p_{4}(k)+14 \phi, q=c-p_{1}(k)-p_{2}(k) \phi-p_{3}(k) \phi^{2}-12 \phi^{3}-14 \phi_{x x}$. 
Review that a regular Sturm-Liouville system has an infinitely many real eigenvalues $\lambda_{0}<\lambda_{1}<\lambda_{2}<\cdots$ with $\lim _{n \rightarrow \infty} \lambda_{n}=\infty$ (see [16]). The eigenfunction $v_{n}(x)$ corresponding to the eigenvalue $\lambda_{n}$ is uniquely determined apart from the different constant factor and has exactly $n$ zeros. Furthermore, via observation we know that $H_{c}$ is a self-adjoint, second order differential operator, hence its eigenvalues $\lambda$ are real and simple. By Weyl's essential spectrum theorem, we have that its essential spectrum is expressed as $\left[c-p_{1}(k), \infty\right)$ owing to the fact that $\lim _{x \rightarrow \infty} q(x)=c-p_{1}(k)$ (see [17]). It can be directly checked that (2.2) is equivalent to $H_{c}\left(\phi_{x}\right)=0$. From the properties of the solitary waves of (2.1), we know that $\phi_{x}$ has exactly one zero on $R$, this indicates that 0 is the second eigenvalue of $H_{c}$. The above analysis leads us to the conclusion that there is exactly one negative eigenvalue, and the rest of the spectrum is positive and bounded away from zero, which shows that assumption (A3) is satisfied.

Secondly, we prove that the scalar function $d(c)$ is convex on a neighborhood of $c$. The following lemma is important in determining the sign of second order derivative of $d(c)$.

Lemma 4.1 ([18]) Set $\Omega=\mathbb{R}$ and let

$$
G_{b}(x)=g_{n}(b) x^{n}+g_{n-1}(b) x^{n-1}+\cdots+g_{1}(b) x+g_{0}(b)
$$

be a family of real polynomials depending also polynomially on a real parameter $b$. Assume that there exists an open interval $I \in \mathbb{R}$ such that:

(i) There is some $b_{0} \in I$ such that $G_{b_{0}}(x)>0$ on $\Omega$.

(ii) For all $b \in I$, the discriminant of $G_{b}$ with respect to $x$ is not equal to zero.

(iii) For all $b \in I, g_{n}(b) \neq 0$.

Then, for all $b \in I, G_{b}(x)>0$ on $\Omega$.

Set

$$
P_{1}(h)=18 h^{5}+75 h^{4}-835 h^{2}-3,000 h-5,250
$$

and

$$
P_{2}(h)=13+10 h+5 h^{2}+6 h^{3} / 5 .
$$

We give our main result as follows.

Theorem 4.2 Let $h^{*}$ be the only zero point of $P_{1}(h)$. If $k=1$ and $13<c<P_{2}\left(h^{*}\right)$, then the scalar function $d(c)$ is convex in a neighborhood of $c$. Therefore, the solitary waves with nonvanishing boundary are orbitally stable when wave speed $c \in\left(13, P_{2}\left(h^{*}\right)\right)$.

Proof Deriving $d(c)$ with respect to $c$, we have

$$
d^{\prime}(c)=\left(c E^{\prime}(\phi)-F^{\prime}(\phi), \phi_{c}\right)+E(\phi)=E(\phi) .
$$


Since $\phi>0$ and $\phi_{x}<0$ in $[0,+\infty)$, it is easy to obtain from (2.4) that

$$
\begin{aligned}
\phi & =-\phi_{x} \sqrt{\frac{c+p_{4}(k)+14 \phi}{c-p_{1}(k)-\frac{p_{2}(k)}{3} \phi-\frac{p_{3}(k)}{6} \phi^{2}-\frac{6}{5} \phi^{3}}} \text { for } x \in(0,+\infty) \\
& =-\phi_{x} \sqrt{\frac{f_{1}(c, k, \phi)}{f_{2}(c, k, \phi)}}
\end{aligned}
$$

where $f_{1}(c, k, \phi)=c+p_{4}(k)+14 \phi, f_{2}(c, k, \phi)=c-p_{1}(k)-\frac{p_{2}(k)}{3} \phi-\frac{p_{3}(k)}{6} \phi^{2}-\frac{6}{5} \phi^{3}$.

Then we calculate the second derivative of $d(c)$,

$$
\begin{aligned}
d^{\prime \prime}(c) & =\frac{\mathrm{d}}{\mathrm{d} c} \int_{R} \frac{1}{2}\left(\phi^{2}+\phi_{x}^{2}\right) \mathrm{d} x \\
& =\frac{\mathrm{d}}{\mathrm{d} c} \int_{0}^{\infty} \phi^{2}\left(1+\frac{f_{2}(c, k, \phi)}{f_{1}(c, k, \phi)}\right) \mathrm{d} x \\
& =-\frac{\mathrm{d}}{\mathrm{d} c} \int_{0}^{\infty} \phi \phi_{x} \sqrt{\frac{f_{1}(c, k, \phi)}{f_{2}(c, k, \phi)}}\left(1+\frac{f_{2}(c, k, \phi)}{f_{1}(c, k, \phi)}\right) \mathrm{d} x
\end{aligned}
$$

Let $h(c)$ denote the amplitude of solitary wave with wave speed $c$, then from (2.4) we know that $h$ is the only positive real number satisfying

$$
c-p_{1}(k)-\frac{p_{2}(k)}{3} h-\frac{p_{3}(k)}{6} h^{2}-\frac{6}{5} h^{3}=0 .
$$

By using transformations $\phi_{x} \mathrm{~d} x=\mathrm{d} y$ and $y=h z,(4.14)$ can be rewritten as

$$
\begin{aligned}
d^{\prime \prime}(c) & =\frac{\mathrm{d}}{\mathrm{d} c} \int_{0}^{h} y \sqrt{\frac{f_{1}(c, k, y)}{f_{2}(c, k, y)}}\left(1+\frac{f_{2}(c, k, y)}{f_{1}(c, k, y)}\right) \mathrm{d} y \\
& =\frac{\mathrm{d}}{\mathrm{d} c} \int_{0}^{1} h^{2} z \sqrt{\frac{f_{1}(c, k, h z)}{f_{2}(c, k, h z)}}\left(1+\frac{f_{2}(c, k, h z)}{f_{1}(c, k, h z)}\right) \mathrm{d} z .
\end{aligned}
$$

Further, substituting $c=c(h)=p_{1}(k)+\frac{p_{2}(k)}{3} h+\frac{p_{3}(k)}{6} h^{2}+\frac{6}{5} h^{3}($ see $(4.15))$ into the above equation, we have

$$
\begin{aligned}
d^{\prime \prime}(c) & =\frac{\mathrm{d}}{\mathrm{d} c} \int_{0}^{1} h^{2} z \sqrt{\frac{f_{1}(c(h), k, h z)}{f_{2}(c(h), k, h z)}}\left(1+\frac{f_{2}(c(h), k, h z)}{f_{1}(c(h), k, h z)}\right) \mathrm{d} z \\
& =\frac{\mathrm{d}}{\mathrm{d} c} \int_{0}^{1} h^{2} z \frac{f_{1}(c(h), k, h z)+f_{2}(c(h), k, h z)}{\sqrt{f_{1}(c(h), k, h z) f_{2}(c(h), k, h z)}} \mathrm{d} z
\end{aligned}
$$

Let $F(h, k, z)$ denote the integrand in (4.17). For any interval $\left[h_{1}, h_{2}\right]$ with $h_{1}>0$ and $\left[k_{1}, k_{2}\right]$ with $k_{1}>0, \int_{0}^{1} F(h, k, z) \mathrm{d} z$ can be regarded as an integral involving parameters. By a direct calculation, we have

$$
\partial_{h} F(h, k, z)=\frac{h^{2} z(1-z) P(h, k, z)}{\left(f_{1}(c(h), k, h z) f_{2}(c(h), k, h z)\right)^{3 / 2}},
$$


where

$$
\begin{aligned}
& f_{1}(c(h), k, h z)=p_{1}(k)+p_{4}(k)+\frac{p_{2}(k)}{3} h+\frac{p_{3}(k)}{6} h^{2}+\frac{6}{5} h^{3}+14 h z, \\
& f_{2}(c(h), k, h z)=h(1-z)\left(\frac{p_{2}(k)}{3}+\frac{p_{3}(k)}{6} h(1+z)+\frac{6}{5} h^{2}\left(1+z+z^{2}\right)\right),
\end{aligned}
$$

and the expression of $P(h, k, z)$ is given as

$$
\begin{aligned}
& P(h, k, z)=\frac{1}{2,700}\left(-186,624 h^{8}-3,265,920 h^{6} z-186,624 h^{8} z-6,350,400 h^{4} z^{2}\right. \\
& -3,265,920 h^{6} z^{2}-18,6624 h^{8} z^{2}-6,350,400 h^{4} z^{3}-3,265,920 h^{6} z^{3} \\
& +93,312 h^{8} z^{3}-6,350,400 h^{4} z^{4}+1,632,960 h^{6} z^{4}+93,312 h^{8} z^{4} \\
& +1,632,960 h^{6} z^{5}+93,312 h^{8} z^{5}+1,632,960 h^{6} z^{6}-233,280 h^{5} p_{1} \\
& -680,400 h^{3} z p_{1}-233,280 h^{5} z p_{1}-680,400 h^{3} z^{2} p_{1}-233,280 h^{5} z^{2} p_{1} \\
& -680,400 h^{3} z^{3} p_{1}+136,080 h^{5} z^{3} p_{1}+136,080 h^{5} z^{4} p_{1}+136,080 h^{5} z^{5} p_{1} \\
& -16,200 h^{2} p_{1}^{2}-16,200 h^{2} z p_{1}^{2}-16,200 h^{2} z^{2} p_{1}^{2}-155,520 h^{6} p_{2} \\
& -1,814,400 h^{4} z p_{2}-77,760 h^{6} z p_{2}-3,528,000 h^{2} z^{2} p_{2}-302,400 h^{4} z^{2} p_{2} \\
& -77,760 h^{6} z^{2} p_{2}-302,400 h^{4} z^{3} p_{2}+77,760 h^{6} z^{3} p_{2}+756,000 h^{4} z^{4} p_{2} \\
& +38,880 h^{6} z^{4} p_{2}+38,880 h^{6} z^{5} p_{2}-129,600 h^{3} p_{1} p_{2}-441,000 h z p_{1} p_{2} \\
& -16,200 h^{3} z p_{1} p_{2}-16,200 h^{3} z^{2} p_{1} p_{2}+64,800 h^{3} z^{3} p_{1} p_{2}-13,500 p_{1}^{2} p_{2} \\
& -43,200 h^{4} p_{2}^{2}-252,000 h^{2} z p_{2}^{2}+84,000 h^{2} z^{2} p_{2}^{2}-3,600 h^{4} z^{2} p_{2}^{2} \\
& +18,000 h^{4} z^{3} p_{2}^{2}-18,000 h p_{1} p_{2}^{2}+7,500 h z p_{1} p_{2}^{2}-4,000 h^{2} p_{2}^{3}+2,000 h^{2} z p_{2}^{3} \\
& -77,760 h^{7} p_{3}-907,200 h^{5} z p_{3}-77,760 h^{7} z p_{3}-1,323,000 h^{3} z^{2} p_{3} \\
& -907,200 h^{5} z^{2} p_{3}-38,880 h^{7} z^{2} p_{3}-1,323,000 h^{3} z^{3} p_{3}-189,000 h^{5} z^{3} p_{3} \\
& +38,880 h^{7} z^{3} p_{3}+415,800 h^{5} z^{4} p_{3}+38,880 h^{7} z^{4} p_{3}+415,800 h^{5} z^{5} p_{3} \\
& +16,200 h^{7} z^{5} p_{3}-64,800 h^{4} p_{1} p_{3}-157,500 h^{2} z p_{1} p_{3}-64,800 h^{4} z p_{1} p_{3} \\
& -157,500 h^{2} z^{2} p_{1} p_{3}-10,800 h^{4} z^{2} p_{1} p_{3}+35,100 h^{4} z^{3} p_{1} p_{3}+35,100 h^{4} z^{4} p_{1} p_{3} \\
& -4,500 h p_{1}^{2} p_{3}-4,500 h z p_{1}^{2} p_{3}-43,200 h^{5} p_{2} p_{3}-252,000 h^{3} z p_{2} p_{3} \\
& -21,600 h^{5} z p_{2} p_{3}-63,000 h^{3} z^{2} p_{2} p_{3}+94,500 h^{3} z^{3} p_{2} p_{3}+17,100 h^{5} z^{3} p_{2} p_{3} \\
& +9,900 h^{5} z^{4} p_{2} p_{3}-18,000 h^{2} p_{1} p_{2} p_{3}-3,750 h^{2} z p_{1} p_{2} p_{3}+8,250 h^{2} z^{2} p_{1} p_{2} p_{3} \\
& -6,000 h^{3} p_{2}^{2} p_{3}+2,250 h^{3} z^{2} p_{2}^{2} p_{3}-10,800 h^{6} p_{3}^{2}-63,000 h^{4} z p_{3}^{2} \\
& -10,800 h^{6} z p_{3}^{2}-63,000 h^{4} z^{2} p_{3}^{2}+26,250 h^{4} z^{3} p_{3}^{2}+5,400 h^{6} z^{3} p_{3}^{2} \\
& +26,250 h^{4} z^{4} p_{3}^{2}+4,050 h^{6} z^{4} p_{3}^{2}-4,500 h^{3} p_{1} p_{3}^{2}-4,500 h^{3} z p_{1} p_{3}^{2} \\
& +2,250 h^{3} z^{2} p_{1} p_{3}^{2}+2,250 h^{3} z^{3} p_{1} p_{3}^{2}-3,000 h^{4} p_{2} p_{3}^{2}-1,500 h^{4} z p_{2} p_{3}^{2} \\
& +1,500 h^{4} z^{2} p_{2} p_{3}^{2}+625 h^{4} z^{3} p_{2} p_{3}^{2}-500 h^{5} p_{3}^{3}-500 h^{5} z p_{3}^{3}+250 h^{5} z^{2} p_{3}^{3} \\
& +250 h^{5} z^{3} p_{3}^{3}-233,280 h^{5} p_{4}-680,400 h^{3} z p_{4}-233,280 h^{5} z p_{4}
\end{aligned}
$$




$$
\begin{aligned}
& -680,400 h^{3} z^{2} p_{4}-233,280 h^{5} z^{2} p_{4}-680,400 h^{3} z^{3} p_{4}+136,080 h^{5} z^{3} p_{4} \\
& +136,080 h^{5} z^{4} p_{4}+136,080 h^{5} z^{5} p_{4}-32,400 h^{2} p_{1} p_{4}-32,400 h^{2} z p_{1} p_{4} \\
& -32,400 h^{2} z^{2} p_{1} p_{4}-129,600 h^{3} p_{2} p_{4}-441,000 h z p_{2} p_{4}-16,200 h^{3} z p_{2} p_{4} \\
& -16,200 h^{3} z^{2} p_{2} p_{4}+64,800 h^{3} z^{3} p_{2} p_{4}-27,000 p_{1} p_{2} p_{4}-18,000 h p_{2}^{2} p_{4} \\
& +7,500 h z p_{2}^{2} p_{4}-64,800 h^{4} p_{3} p_{4}-157,500 h^{2} z p_{3} p_{4}-64,800 h^{4} z p_{3} p_{4} \\
& -157,500 h^{2} z^{2} p_{3} p_{4}-10,800 h^{4} z^{2} p_{3} p_{4}+35,100 h^{4} z^{3} p_{3} p_{4} \\
& +35,100 h^{4} z^{4} p_{3} p_{4}-9,000 h p_{1} p_{3} p_{4}-9,000 h z p_{1} p_{3} p_{4}-18,000 h^{2} p_{2} p_{3} p_{4} \\
& -3,750 h^{2} z p_{2} p_{3} p_{4}+8,250 h^{2} z^{2} p_{2} p_{3} p_{4}-4,500 h^{3} p_{3}^{2} p_{4}-4,500 h^{3} z p_{3}^{2} p_{4} \\
& +2,250 h^{3} z^{2} p_{3}^{2} p_{4}+2,250 h^{3} z^{3} p_{3}^{2} p_{4}-16,200 h^{2} p_{4}^{2}-16,200 h^{2} z p_{4}^{2} \\
& \left.-16,200 h^{2} z^{2} p_{4}^{2}-13,500 p_{2} p_{4}^{2}-4,500 h p_{3} p_{4}^{2}-4,500 h z p_{3} p_{4}^{2}\right),
\end{aligned}
$$

where $p_{i}=p_{i}(k)(i=1,2,3,4)$.

From (4.18) we know that there exists a positive constant $K$ related to $\left[h_{1}, h_{2}\right]$ and $\left[k_{1}, k_{2}\right]$ such that

$$
\left|\partial_{h} F(h, k, z)\right| \leq K(1-z)^{-\frac{1}{2}} \quad \text { for all }(h, k, z) \in\left[h_{1}, h_{2}\right] \times\left[k_{1}, k_{2}\right] \times(0,1)
$$

Set $g(z)=K(1-z)^{-1 / 2}$, then $g(z) \in L^{1}(0,1)$. By the dominated convergence theorem, we have

$$
\partial_{h} \int_{0}^{1} F(h, k, z) \mathrm{d} z=\int_{0}^{1} \partial_{h} F(h, k, z) \mathrm{d} z
$$

for all $h \in\left[h_{1}, h_{2}\right]$ and $k \in\left[k_{1}, k_{2}\right]$. Therefore we can extend this result to all $h \in(0,+\infty)$ and $k \in(0,+\infty)$, with the monotonic increase of amplitude $h$ with wave speed $c$, we have

$$
d^{\prime \prime}(c)=\int_{0}^{1} \partial_{h} F(h, k, z) \mathrm{d} z \cdot h^{\prime}(c)
$$

where $h^{\prime}(c)>0$ denotes the derivative of amplitude $h$ with respect to wave speed $c$.

It is observed from (4.18) and (4.21) that the sign of $d^{\prime \prime}(c)$ is determined by $P(h, k, z)$, but it is difficult to judge the sign of it. So we take a step back to consider the special case $k=1$, then (4.18) is simplified as

$$
\partial_{h} F(h, z)=\frac{h^{2} z(1-z) P(h, z)}{\left(f_{1}(c(h), h z) f_{2}(c(h), h z)\right)^{3 / 2}},
$$

where

$$
\begin{aligned}
& f_{1}(c(h), h z)=28+10 h+5 h^{2}+\frac{6}{5} h^{3}+14 h z, \\
& f_{2}(c(h), h z)=h(1-z)\left(10+5 h(1+z)+\frac{6}{5} h^{2}\left(1+z+z^{2}\right)\right),
\end{aligned}
$$


and

$$
\begin{aligned}
P(h, z)= & \frac{1}{125}\left(1,470,000+840,000 h+233,800 h^{2}-21,000 h^{4}-5,040 h^{5}\right. \\
& +3,080,000 h z+1,708,800 h^{2} z+601,200 h^{3} z+126,000 h^{4} z+12,960 h^{5} z \\
& +2,473,800 h^{2} z^{2}+1,141,200 h^{3} z^{2}+359,650 h^{4} z^{2}+74,210 h^{5} z^{2}+11,250 h^{6} z^{2} \\
& +900 h^{7} z^{2}+1,180,200 h^{3} z^{3}+470,400 h^{4} z^{3}+146,390 h^{5} z^{3}+33,300 h^{6} z^{3} \\
& +5,400 h^{7} z^{3}+432 h^{8} z^{3}+380,275 h^{4} z^{4}+116,640 h^{5} z^{4}+29,835 h^{6} z^{4} \\
& +5,400 h^{7} z^{4}+432 h^{8} z^{4}+75,390 h^{5} z^{5}+12,960 h^{6} z^{5}+2,250 h^{7} z^{5} \\
& \left.+432 h^{8} z^{5}+7,560 h^{6} z^{6}\right),
\end{aligned}
$$

where $(h, z) \in(0, \infty) \times(0,1)$. The transformation $z=\frac{x^{2}}{1+x^{2}}$ can be used to map the variable $z$ from $(0,1)$ to the whole real line $\mathbb{R}$, then we use Lemma 4.1 to determine the sign of polynomial $P(h, z)$ with parameter $h$.

Substituting $z=\frac{x^{2}}{1+x^{2}}$ into (4.25), we have

$$
\begin{aligned}
P_{h}(x)= & \frac{1}{125\left(1+x^{2}\right)^{6}}\left(1,470,000+840,000 h+233,800 h^{2}-21,000 h^{4}-5,040 h^{5}\right. \\
& +\left(8,820,000+8,120,000 h+3,111,600 h^{2}+601,200 h^{3}-17,280 h^{5}\right) x^{2} \\
& +\left(22,050,000+28,000,000 h+14,524,800 h^{2}+4,147,200 h^{3}+674,650 h^{4}\right. \\
& \left.+63,410 h^{5}+11,250 h^{6}+900 h^{7}\right) x^{4} \\
& +\left(29,400,000+47,600,000 h+31,659,200 h^{2}+11,757,000 h^{3}\right. \\
& \left.+2,749,000 h^{4}+472,030 h^{5}+78,300 h^{6}+9,000 h^{7}+432 h^{8}\right) x^{6} \\
& +\left(22,050,000+43,400,000 h+35,437,800 h^{2}+16,399,800 h^{3}\right. \\
& \left.+4,894,375 h^{4}+1,055,070 h^{5}+197,235 h^{6}+27,000 h^{7}+1,728 h^{8}\right) x^{8} \\
& +\left(8,820,000+20,440,000 h+19,842,000 h^{2}+11,111,400 h^{3}+4,114,350 h^{4}\right. \\
& \left.+1,079,240 h^{5}+217,530 h^{6}+32,850 h^{7}+2,592 h^{8}\right) x^{10} \\
& +\left(1,470,000+3,920,000 h+4,416,400 h^{2}+2,922,600 h^{3}+1,315,325 h^{4}\right. \\
& \left.\left.+420,550 h^{5}+94,905 h^{6}+13,950 h^{7}+1,296 h^{8}\right) x^{12}\right) .
\end{aligned}
$$

Set $P_{h}(x)=\frac{N_{h}(x)}{D(x)}$, obviously the denominator $D(x)$ in $P_{h}(x)$ is absolutely greater than zero. We need to prove that the numerator $N_{h}(x)$ is over zero, which can be treated as a one-parametric family of polynomials with parameter $h \in(0, \infty)$ and can be handled by Lemma 4.1. With the help of Maple or Mathematica, the discriminant of $N_{h}(x)$ can be obtained as

$$
\begin{aligned}
\Delta= & -974,098,582,732,800,000 h^{60}\left(-5,250-3,000 h-835 h^{2}+75 h^{4}+18 h^{5}\right) \\
& \times\left(58,800+30,100 h+10,825 h^{2}+2,040 h^{3}+2,415 h^{4}+900 h^{5}+108 h^{6}\right)^{4} \\
& \times\left(1,470,000+3,920,000 h+4,416,400 h^{2}+2,922,600 h^{3}\right.
\end{aligned}
$$




$$
\begin{aligned}
& \left.+1,315,325 h^{4}+420,550 h^{5}+94,905 h^{6}+13,950 h^{7}+1,296 h^{8}\right) \\
& \times(10,527,552,696,056,300,836,353,072,000,000,000,000 \\
& +39,306,760,598,215,044,821,831,490,000,000,000,000 h \\
& +75,333,718,338,168,614,240,153,258,910,000,000,000 h^{2} \\
& \text { + 99,618,621,855,054,289,426,838,010,255,000,000,000h }{ }^{3} \\
& +102,779,684,628,263,794,779,502,999,420,625,000,000 h^{4} \\
& +88,249,916,544,459,627,952,310,998,095,312,500,000 h^{5} \\
& +65,303,345,107,623,498,315,297,763,904,460,937,500 h^{6} \\
& +42,548,078,554,608,716,720,415,000,636,792,968,750 h^{7} \\
& +24,860,972,299,032,069,597,285,337,353,435,937,500 h^{8} \\
& +13,275,168,601,838,580,408,774,342,743,598,046,875 h^{9} \\
& +6,584,544,306,510,110,821,769,951,522,397,265,625 h^{10} \\
& +3,061,087,654,026,717,393,086,768,379,532,656,250 h^{11} \\
& +1,335,781,177,106,125,623,220,777,826,553,984,375 h^{12} \\
& +546,581,319,914,479,884,670,825,019,152,734,375 h^{13} \\
& +209,902,282,786,161,951,852,852,247,181,906,250 h^{14} \\
& +75,835,942,043,197,348,513,832,530,299,890,625 h^{15} \\
& +25,790,280,220,863,758,433,223,640,939,343,750 h^{16} \\
& +8,230,241,786,176,630,163,097,372,845,306,250 h^{17} \\
& +2,452,965,279,312,714,362,680,952,709,421,875 h^{18} \\
& +680,524,665,454,209,261,845,696,176,096,875 h^{19} \\
& +175,481,686,500,525,607,025,019,806,094,375 h^{20} \\
& +41,967,547,117,323,743,984,292,464,355,000 h^{21} \\
& +9,253,162,584,359,279,323,694,970,356,250 h^{22} \\
& +1,860,515,408,068,033,078,135,476,990,000 h^{23} \\
& +336,138,021,290,024,276,087,312,149,500 h^{24} \\
& +53,620,961,897,451,577,435,040,772,000 h^{25} \\
& +7,399,996,680,369,897,120,613,849,200 h^{26} \\
& +861,847,511,676,410,348,045,222,400 h^{27} \\
& +82,038,790,520,521,056,364,723,200 h^{28} \\
& +6,107,780,869,054,049,060,536,320 h^{29} \\
& +332,825,048,384,446,232,248,320 h^{30} \\
& +11,808,121,415,612,085,043,200 h^{31} \\
& \left.+205,521,196,952,300,027,904 h^{32}\right)^{2} \text {. }
\end{aligned}
$$


We take $h_{0}=1$ to get that

$$
\begin{aligned}
N_{1}(x)= & 2,517,760+20,635,520 x^{2}+69,472,210 x^{4}+123,724,962 x^{6}+123,463,008 x^{8} \\
& +65,659,962 x^{10}+14,575,026 x^{12}>0
\end{aligned}
$$

which ensures the assumption (i) in Lemma 4.1. We write (4.27) simply as

$$
\Delta=-P_{1}(h) P^{+}(h)
$$

where $P_{1}(h)$ see $(4.10)$ and $P^{+}(h)>0$ consists of the remainder of $\Delta$. From the expression of $P_{1}(h)$ we know that it has only one real zero $h^{*}$ which lies in the interval $(3,4)$ and $P_{1}(h)<0$ as $h \in\left(0, h^{*}\right)$, namely the discriminant (4.29) of $N_{h}(x)$ is positive, which ensures that the assumption (ii) in Lemma 4.1 is satisfied.

Moreover, for all $h \in\left(0, h^{*}\right)$, the coefficient of the most order term $x^{12}$ in $N_{h}(x)$ is positive, which guarantees the assumption (iii) in Lemma 4.1.

When wave amplitude $h \in\left(0, h^{*}\right)$, the corresponding wave speed $c \in\left(13, P_{2}\left(h^{*}\right)\right)$, where $P_{2}(h)$ see (4.11). Therefore, in summary, we have $d^{\prime \prime}(c)>0$, this completes the proof of Theorem 4.2.

\footnotetext{
Acknowledgements

This work is partially supported by the National Natural Science Foundations of China (Grant No. 11401096), (Grant No. 11526062) and (Grant No. 51608119). The authors thank the editors for their hard work and also gratefully acknowledge helpful comments and suggestions by the reviewers.
}

\section{Competing interests}

The authors declare that they have no competing interests.

\section{Authors' contributions}

The authors contributed equally to this paper. All authors read and approved the final manuscript.

\section{Author details}

'Department of Basic Courses, Guangzhou Maritime Institute, Guangzhou, 510725, P.R. China. ${ }^{2}$ Department of Mathematics, Foshan University, Guangdong, 528000, P.R. China.

\section{Publisher's Note}

Springer Nature remains neutral with regard to jurisdictional claims in published maps and institutional affiliations.

Received: 14 March 2017 Accepted: 11 May 2017 Published online: 23 May 2017

\section{References}

1. Constantin, A, Lannes, D: The hydrodynamical relevance of the Camassa-Holm and Degasperis-Procesi equations Arch. Ration. Mech. Anal. 192, 165-186 (2009)

2. Johnson, RS: Camassa-Holm, Korteweg-de Vries and related models for water waves. J. Fluid Mech. 455, 63-82 (2002)

3. $\mathrm{Mi}$, YS, Mu, CL: On the solutions of a model equation for shallow water waves of moderate amplitude. J. Differ. Equ. $255,2101-2129(2013)$

4. Lai, SY, Wu, YH: The local well-posedness and existence of weak solutions for a generalized Camassa-Holm equation. J. Differ. Equ. 248, 2038-2063 (2010)

5. Kato, T: Quasi-linear equations of evolution with application to partial differential equations. In: Spectral Theory and Differential Equations, pp. 25-70. Springer, Berlin (1975)

6. Duruk Mutlubas, N: On the Cauchy problem for a model equation for shallow water waves of moderate amplitude. Nonlinear Anal., Real World Appl. 14, 2022-2026 (2013)

7. Duruk Mutlubas, N: Local well-posedness and wave breaking results for periodic solutions of a shallow water equation for waves of moderate amplitude. Nonlinear Anal. 97, 145-154 (2014)

8. Zhou, SM, Mu, CL: Global conservative solutions for a model equation for shallow water waves of moderate amplitude. J. Differ. Equ. 256, 1793-1816 (2014)

9. Geyer, A: Solitary traveling water waves of moderate amplitude. J. Nonlinear Math. Phys. 19, 104-115 (2012)

10. Duruk Mutlubas, N, Geyer, A: Orbital stability of solitary waves of moderate amplitude in shallow water. J. Differ. Equ. $255,254-263(2013)$ 
11. Grillakis, M, Shatah, J, Strauss, W: Stability theory of solitary waves in the presence of symmetry I. J. Funct. Anal. 74, 160-197 (1987)

12. Constantin, A, Strauss, W: Stability of the Camassa-Holm solitons. J. Nonlinear Sci. 12, 415-422 (2002)

13. Gasull, A, Geyer, A: Traveling surface waves of moderate amplitude in shallow water. Nonlinear Anal. 102, 105-119 (2014)

14. Benjamin, TB: The stability of solitary waves. Proc. R. Soc. Lond. A 328, 153-183 (1972)

15. Benjamin, TB, Bona, JB, Mahony, JJ: Model equations for long waves in nonlinear dispersive systems. Philos. Trans. R. Soc. Lond. Ser. A 272, 47-78 (1972)

16. Birkhoff, G, Rota, GC: Ordinary Differential Equations. Wiley, New York (1998)

17. Dunford, N, Schwarz, JT: Linear Operators, Part II: Spectral Theory. Wiley-Interscience, New York (1963)

18. Garca-Saldaa, JD, Gasull, A, Giacomini, H: Bifurcation values for a family of planar vector fields of degree five. Discrete Contin. Dyn. Syst. 35(2), 669-701 (2012)

Submit your manuscript to a SpringerOpen ${ }^{\circ}$ journal and benefit from:

- Convenient online submission

Rigorous peer review

- Immediate publication on acceptance

- Open access: articles freely available online

- High visibility within the field

- Retaining the copyright to your article 